Received: February 19, 2020

Accepted: February 25, 2020

Correspondence

Saverio Comitini

UOC Ortopedia e Traumatologia, Ospedale Maggio-

re AUSL Bologna, Largo Nigrisoli 2,

40133 Bologna, Italy

E-mail: saveriocomitini@gmail.com

English proofreading by Ms. Enza Scifo

Conflict of interest

The Authors declare no conflict of interest

How to cite this article: Comitini S, Berti M, Ganci M, et al. Outcome of humeral shaft fractures. Lo Scalpello 2020;34:9-20. https://doi. org/10.36149/0390-5276-002

(C) Ortopedici Traumatologi Ospedalieri d'Italia (O.T.O.D.i.) 2020

\section{(c) (1) (2) $\bigcirc$}

This is an open access article distributed in accordance with the CC-BY-NC-ND (Creative Commons Attribution-NonCommercial-NoDerivatives 4.0 International) license. The article can be used by giving appropriate credit and mentioning the license, but only for non-commercial purposes and only in the original version. For further information: https://creativecommons.org/licenses/by-nc-nd/4.0/deed.en

\title{
Outcome of humeral shaft fractures
}

\author{
Saverio Comitini', Matteo Berti², Marco Ganci ${ }^{3}$, \\ Luca Amendola', Paolo Barca', Emanuela Castiello', \\ Matteo Commessatti', Guido Grippo', Domenico S. Tigani' \\ ${ }^{1}$ Unità operativa di Ortopedia e Traumatologia, Ospedale Maggiore C. A. Pizzardi, \\ Bologna, Italy; ${ }^{2}$ Università degli studi di Bologna, IRCCS Istituto Ortopedico Rizzoli, \\ Bologna, Italy; ${ }^{3}$ Università degli studi di Catania, Azienda Ospedaliero-Universitaria \\ Policlinico, Vittorio Emanuele, Catania, Italy
}

\begin{abstract}
SUMMARY
Humeral shaft fractures represent approximately $3 \%$ of all long-bone fractures and have historically been treated successfully in a non-operative way, but surgical management is indicated in several conditions. Although there are numerous randomized clinical trials and meta-analyses that have attempted to guide surgeons, there is little evidence and no consensus as to the most suitable treatment. Every treatment has its advantages and its complications, and many factors must be considered: not only the features of the fracture, but also patient compliance and surgeon experience.
\end{abstract}

Key words: humeral shaft, fractures, treatment, radial nerve palsy, outcome

\section{Epidemiology}

Fractures of the humerus are common, comprising approximately 5 to $8 \%$ of all extremity fractures. Humeral shaft fractures account for approximately $3 \%$ of all long-bone fractures ${ }^{1}$. The overall annual incidence is 14.5 per 100,000 per year, with a bimodal age distribution: the first peak occurs in the third decade and consists mostly of high-energy trauma male patients, and a second larger peak occurs in the eighth decade and consists mostly of low-energy female patients sustaining a simple ground level fall ${ }^{2,3}$. Humeral shaft fractures are also a marker of severe injury in trauma patients and carry a high mortality rate $^{4}$.

\section{Classification}

Humeral shaft extends from the proximal border of pectoralis major insertion to the supracondylar ridge and fractures can be classified according to the Orthopaedic Trauma Association (OTA)/Arbeitsgemeinschaft für Osteosynthesefragen (AO) combined classification ${ }^{5}$ as AO 12-:

\footnotetext{
- A (simple):

- spiral

- oblique $\left(\geq 30^{\circ}\right)$

- transverse $\left(<30^{\circ}\right)$

- $\quad$ B (wedge):

- intact wedge

- fragmentary wedge
} 
- $\mathbf{C}$ (complex-multifragmentary):

- intact segment

- fragmentary segment

\section{Non-operative treatment}

The preferred treatment for humeral shaft fractures has historically been non-operative management. In 1977, Sarmiento et al. described conservative treatment with a functional, moldable splint which allowed early return to activity, acceptable functional outcomes and minimal morbidity ${ }^{6-8}$. This treatment has been shown to achieve an excellent or good result in nearly $94.5 \%$ of cases with a mean time of healing of 10.7 weeks, so it has been widely accepted as the gold standard for treating humeral shaft fractures conservatively ${ }^{9}$.

Complications of non-operative management include nonunion, malunion, persistent radial nerve deficits and joint stiffness. Major reasons for failed conservative treatment are an incorrect indication, a significant deformity and a transverse fracture pattern ${ }^{7}$.

Delayed union or nonunion, defined as radiographic detection of delayed consolidation after 6-8 months from treatment, leads to discomfort, pain and limited function of the upper extremity ${ }^{10}$. Transverse fracture patterns might be more prone to nonunion than oblique/spiral patterns due to less bone contact area, and bracing does not permit compression of the fracture site. In contrast, muscle and soft tissue invagination into the fracture site in oblique/spiral fractures can be the cause of nonunion while in transverse fractures it is uncommon ${ }^{1,11}$.

Humerus is not a load bearing bone so that the deformity can be tolerated. In 1966, Klenerman reviewed 32 patients with humeral shaft fractures and noted that $20^{\circ}$ in procurvatum or $30^{\circ}$ of varus were the limits for the deformities to become clinically relevant. These values continue to be adopted as the acceptable radiographic parameters for bracing, including the addition of $30^{\circ}$ of acceptable valgus deformity, $15^{\circ}$ of acceptable rotational deformity and acceptable shortening of less than $3 \mathrm{~cm}^{1,12}$.

Persistent radial nerve deficits are due to the obvious impossibility of surgical exploration with conservative treatment, while elbow stiffness can be favored by a long period of brace immobilization.

Patients with a significant psychiatric history had particularly poor outcomes with conservative management, probably due to poorer compliance to treatment ${ }^{13}$ (Figs. 1A, B, C).

The decision to use functional bracing in polytrauma patients should depend on the time of expected immobilization, on the presence of additional fractures of the ipsilateral upper extremity and on the patient's need for crutches ${ }^{14}$.
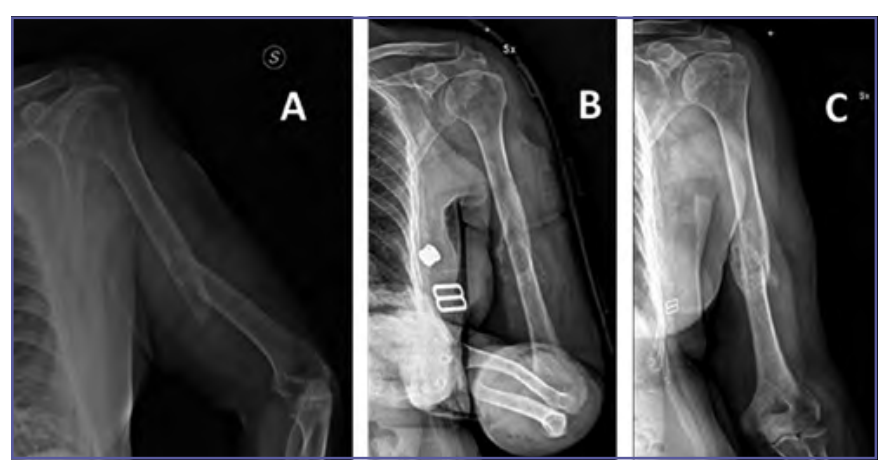

Figure 1. Clinical Case 1. AO 12A1 female 76y with high pre-operative risk. A) post-trauma X-ray; B) X-ray during conservative treatment with brace 45 days after the trauma; C) X-ray 5 months after the trauma.

\section{Operative treatment}

Surgical treatment is usually reserved when conservative treatment fails (irreducible fracture or unacceptable reduction, nonunion or malunion), for open fractures that require debridement and stabilization, in cases of neurovascular injury, in polytrauma, in fractures extended into the joints, in floating elbow and in patients who request the possibility of early mobilization ${ }^{15,16}$.

The ultimate goal of fixation is rigid stabilization to allow early range of motion, protection of the neurovascular structures and preservation of the tricep function posteriorly and the elbow flexor muscles anteriorly ${ }^{17,18}$, so knowledge of the complex neurovascular anatomy of the arm is imperative to accomplish a safe surgical approach.

Radial nerve injury after humeral shaft fractures has an overall incidence of $11.8 \%$, representing the most common peripheral nerve injury associated with bone fractures ${ }^{1,19}$. This high percentage is attributable to the intimate contact of the radial nerve with the periosteum of the humerus. In particular, it was found to be in direct contact with the posterior humerus from $17.1 \pm 1.6 \mathrm{~cm}$ to $10.9 \pm 1.5 \mathrm{~cm}$ proximal to the lateral epicondyle, while distally the nerve coursed anterior to the humerus and became protected by brachialis muscle at the level of the proximal aspect of the lateral metaphyseal flare. Thus, the risk of nerve lesion is higher in two sites, defined danger zones: at the posterior mid-shaft, where the nerve lies in contact with the humerus, and at the distal lateral humerus as it pierces the lateral intermuscular septum ${ }^{20}$.

Early surgical exploration must be recommended in several cases such as associated vascular or severe soft tissue injury, radial nerve deficit after manipulation (secondary nerve palsy), intractable neurogenic pain suggesting nerve entrapment or compression and high suspicion of nerve laceration with spiral oblique fractures. Outside of these recommendations, expectant observation is recommended due to high rate 
of spontaneous recovery of the radial nerve after closed humeral shaft fractures ${ }^{19,21,22}$.

\section{Open reduction and plate fixation}

Plate osteosynthesis allows anatomical reduction, direct viewing of the fracture site, interfragmentary compression and the chance to explore and isolate the radial nerve ${ }^{15}$.

Adhering to basic AO principles of fracture management is important when plate fixation is performed ${ }^{10}$. Plates can be used for direct compression fracture fixation, neutralization of lag screw interfragmentary fixation or in a bridging fashion. Direct reduction with absolute stability fixation is ideal when the morphology of the fracture allows it ${ }^{1}$.

Compression plating seems to be the best method of treatment of humeral fracture nonunion, with advantages of stable fixation, compression of the bone stumps, protection of neurovascular elements, sparing of shoulder and elbow joint from injuries and stiffness. At the same time, direct access to nonunion site leads to resect nonunion, removing of all fibrous tissues and drilling the canal enhancing local biology ${ }^{10}$ (Figs. 2A, B, C; Figs. 3A, B, C, D, E, F).

Plate fixation has the main disadvantage of extensive surgical dissection leading to iatrogenic injuries, such as soft tissue stripping or radial nerve damage. With the improvement of plating techniques and increasing incidence in plating management of unstable humeral shaft fractures, iatrogenic injuries to the radial nerve have been reported in 4.2 to $5.1 \%$ of cases. Multiple approaches to the posterior humerus have been
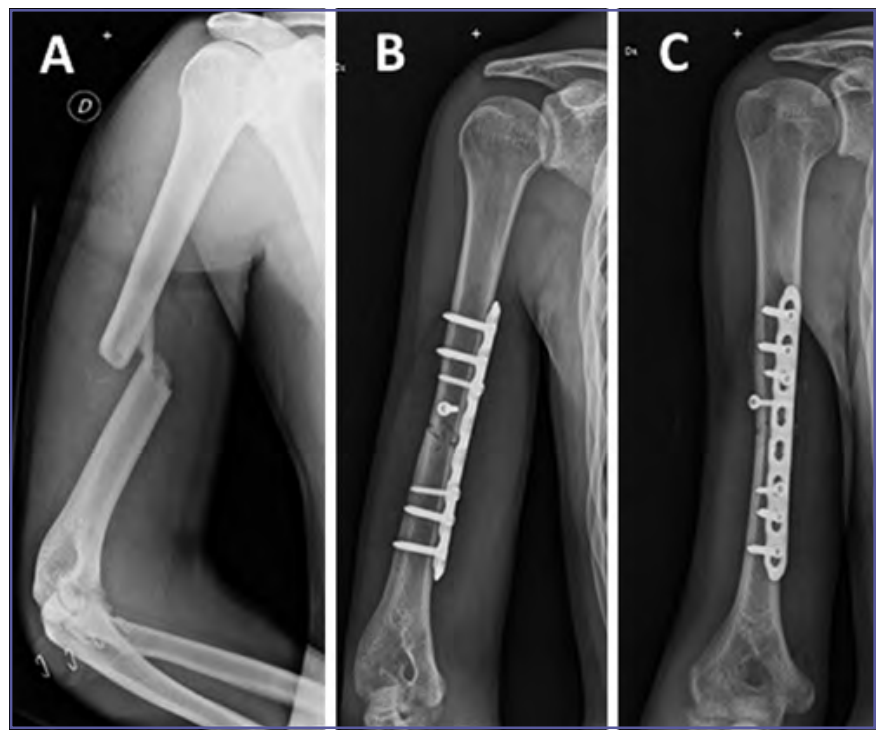

Figure 2. Clinical Case 2. AO 12B2 male 22y. Interfragmentary screw and compressive plate osteosynthesis. A) pre-operative X-ray; B-C) post-operative X-rays at one month. described, including the anterior, anterolateral, lateral, posterior and modified posterior: radial nerve is at considerable risk in each of these approaches. Its dentification during the surgical approach allows for protection and aids in its identification in the event of a future revision surgery ${ }^{17,23,24}$.

Gerwin et al. found that the radial nerve traversed the posterior humerus $20.7 \pm 1.2 \mathrm{~cm}$ proximal to the medial epicondyle to $14.2 \pm 0.6 \mathrm{~cm}$ proximal to the lateral epicondyle ${ }^{25}$. This reference, although helpful in identification of the radial nerve's path and useful for pre-operative planning, may not be as useful when addressing comminuted fractures, malunion/ nonunion and other pathologic conditions that cause alteration of humeral anatomy. Identification of the point of confluence between the long and lateral heads of the triceps and the triceps aponeurosis provides an easily identifiable superficial landmark along the posterior approach that can be considered adjunctive and complementary to previously cited method by Gerwin ${ }^{24}$ (Figs. 4A, B, C, D).

Nevertheless, anatomical reduction and correct osteosynthesis are not an absolute guarantee of healing because there is no certainty of consolidation. Excessive fragment detachment, unfortunately, is frequently unavoidable in complex fractures to achieve an anatomical reduction, leading to bone resorption and nonunion. Factors that lead to a fixation failure are comminution, open fractures and mechanical instability of the implant ${ }^{10}$.

\section{Intramedullary nailing}

Although several studies have compared the clinical outcomes of plating versus intramedullary nailing (IMN) in the treatment of humeral shaft fractures, the optimal surgical treatment remains controversial ${ }^{26-28}$.

Hongjie Wen et al. in 2019 performed a meta-analysis to compare the efficacy and safety between antegrade IMN and plating for humeral shaft fracture, concluding that IMN may be superior to open reduction and internal fixation (ORIF) in reducing blood loss and post-operative infections, but inferior to minimally invasive plate osteosynthesis (MIPO) in nonunion rate. In the same study they found no statistically significant differences in operation time, functional results (evaluated with American Shoulder and Elbow Surgeons (ASES) score), rate of nerve injury, delayed union and need for reoperation ${ }^{27}$ (Figs. 5A, B; Figs. 6A, B, C, D, E, F).

Nail introduction through the rotator cuff may create irreversible damage and possible limitation of shoulder mobility due to partial or complete tear of the rotator cuff (supraspinatus tendon is the most frequently involved ${ }^{29}$ ), sub-acromial bursitis or not specific inflammatory changes of the acromioclavicular joint ${ }^{30}$. Nevertheless, recent studies have demonstrated the safety of this technique, probably due to an increasing sensibilization about the problem and the consequent use of a more cautious transversal proximal surgical access followed 


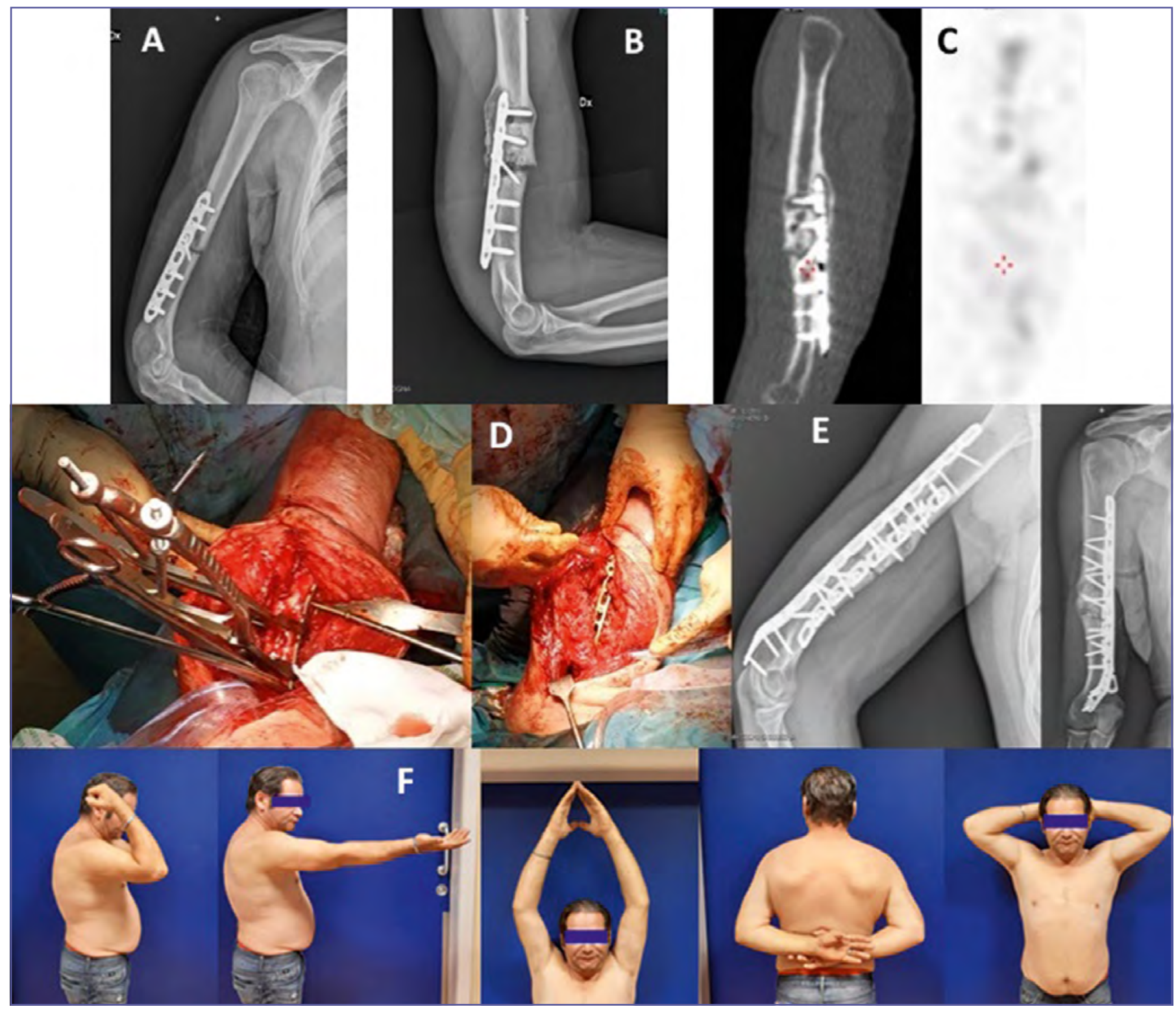

Figure 3. Clinical Case 3. AO 12A3 male 39y patient treated in another hospital with inadequate osteosynthesis that led to a nonunion. A) X-ray 3 months after operative treatment; B) X-rays after 18 months after surgery; C) Negative radiolabeled WBC scintigraphy and inflammatory indexes ruled out the possibility of infection; D) Posterior surgical access and double plate osteosynthesis of the nonunion site; E) X-ray 3 months after the surgery; F) three months follow-up showed good clinical and functional recovery.

by an accurate rotator cuff tendon reconstruction at the end of the procedure ${ }^{15,29}$ (Figs. 7A, B, C, D).

An alternative to antegrade nailing is the use of an elastic intramedullary nail, such as Marchetti-Vicenzi nail, which, with a retrograde insertion, has proven to be useful and safe for shoulder function and, at the same time, allows a solid proximal fixation by means of a bundle of divergent pins ${ }^{31}$ (Figs. 8A, B, C, D; Figs. 9A, B; Figs. 10A, B).

\section{External fixation}

The main indications for the use of external fixation in diaphyseal humeral fractures are open fractures, polytrauma, patients with severe soft tissues problems, gunshot wounds and pediatric fractures ${ }^{15}$. In most cases an external fixator should be considered as a temporary treatment in following the principles of damage control orthopedics (DCO), but in 


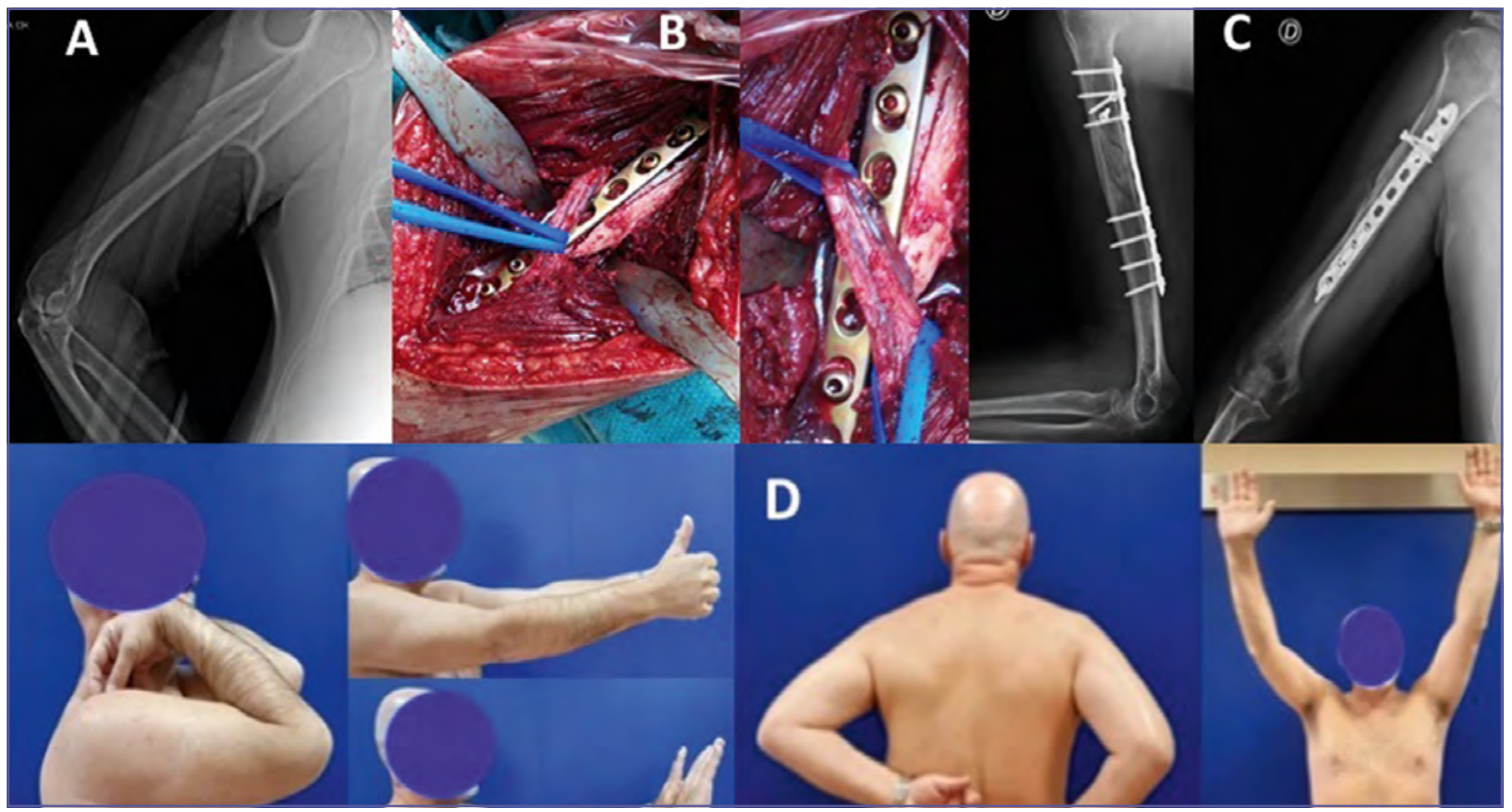

Figure 4. Clinical Case 4. AO $12 \mathrm{C} 1$ male 55y. post-traumatic radial nerve stupor. Trans-tricipital posterior access allowed radial nerve exploration and protection at the fracture site before plating. A) pre-operative $X$-ray; $B$ ) intraoperative photos show osteosynthesis with plate and radial nerve isolated; C) post-operative $\mathrm{X}$-ray 1 month after surgery; D) clinical photos show functional recovery 3 months after the trauma.

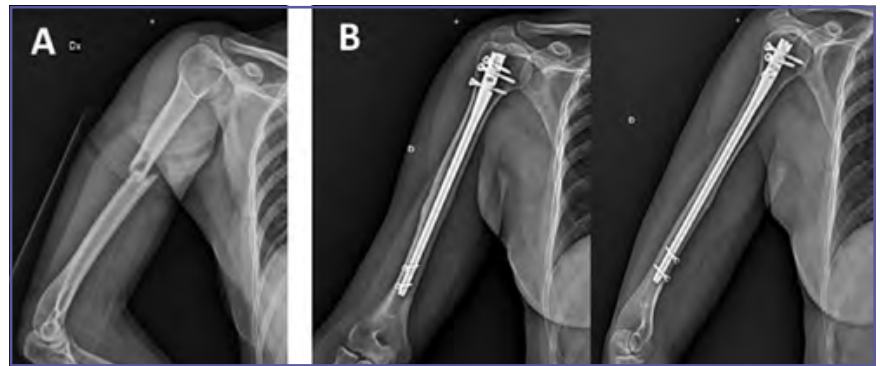

Figure 5. Clinical Case 5. AO 12A3 female 44y. Anterograde intramedullary nail osteosynthesis with good reduction and healing of the fracture site. A) pre-operative X-ray; B) X-ray after 17 months after trauma show radiological healing.

selected cases it can be performed as definitive treatment with good results ${ }^{32}$.

External fixation is the most rapid and minimally invasive technique compared to intramedullary splint and to plate synthesis and it doesn't cause lesions to rotator cuff or elbow joint. Another advantage is mainly due to fracture hematoma retention, which is not possible with open reduction techniques ${ }^{15}$. One of the main features that differentiates external fixation from other methods of treatment is its ability to ensure adequate stability without excessive rigidity, which is crucial as biological stimulus for good healing of the fracture ${ }^{15}$, but adequate stability is not always easy to obtain with miniinvasive monoaxial external fixators.

Long time of healing (due to dynamic and not anatomical fracture reduction), system encumbrance and the necessity of periodical medications can significantly limit patient compliance.

As for intramedullary nailing, close reduction does not give the possibility to explore the radial nerve that, at the same time, is at risk of injury during distal pin insertion (Figs. 11A, B, C, D, E).

\section{Complications: radial nerve palsy}

The intimate contact between the radial nerve and humeral diaphysis makes it particularly vulnerable to traction, stretching or entrapment following fracture of the middle-distal third of the humerus.

The incidence of radial nerve palsy (RNP) after a humeral shaft fracture has been estimated to be between 7 and 17\%, making it the most common nerve lesion complicating long bone fractures ${ }^{33}$. 


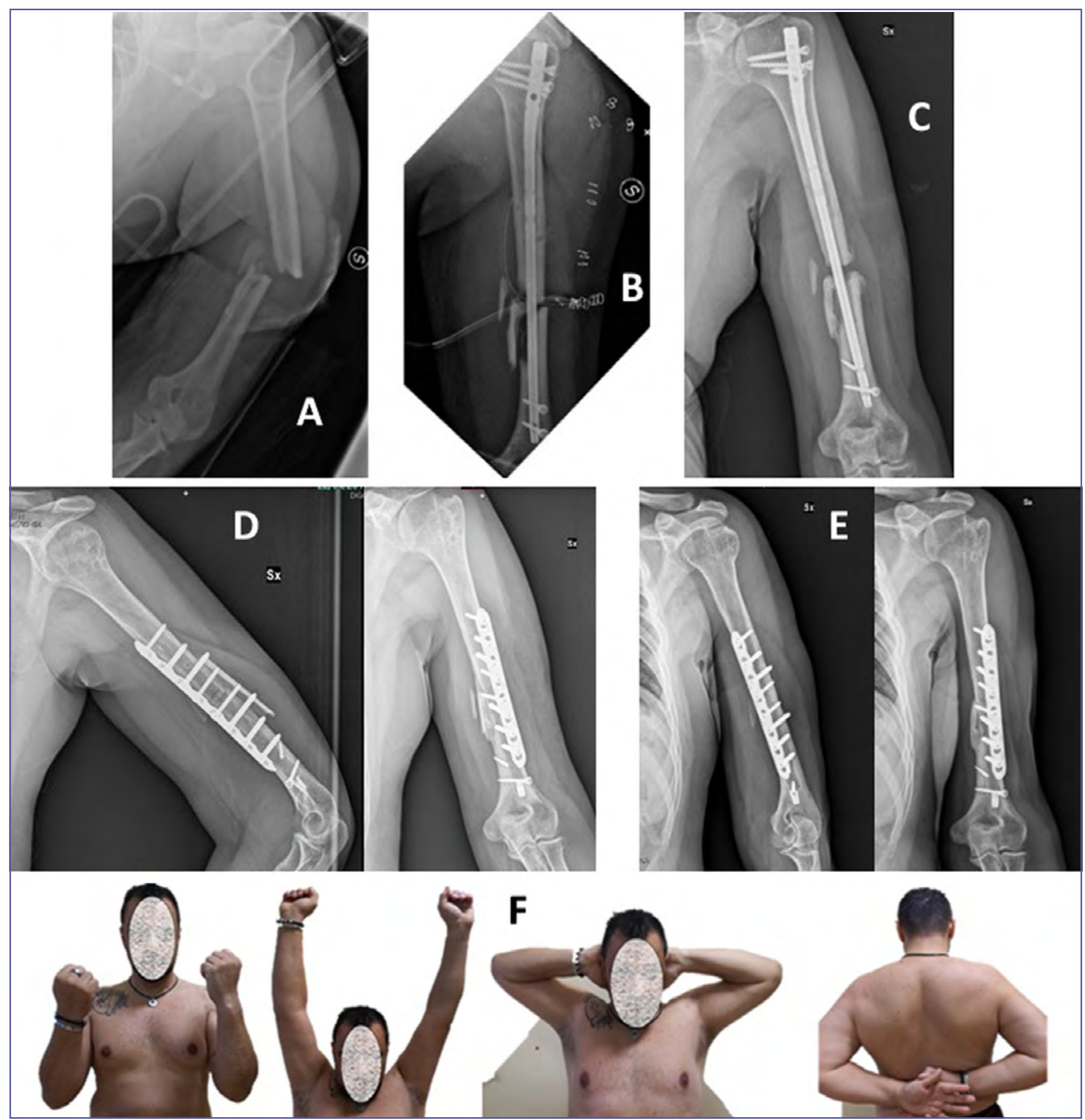

Figure 6. Clinical Case 6. AO 12B3 male 34y polytrauma. Intramedullary nail fixation without compression on fracture side led to nonunion. A) pre-operative X-ray; B) X-ray post-operative treatment; C) X-ray 1 year after trauma show nonunion; D) revision with compression plate and opposite bone graft; E) X-ray 1 year after revision show radiological healing of the nonunion; F) clinical photos 6 months after revision surgery show very good functional recovery. 

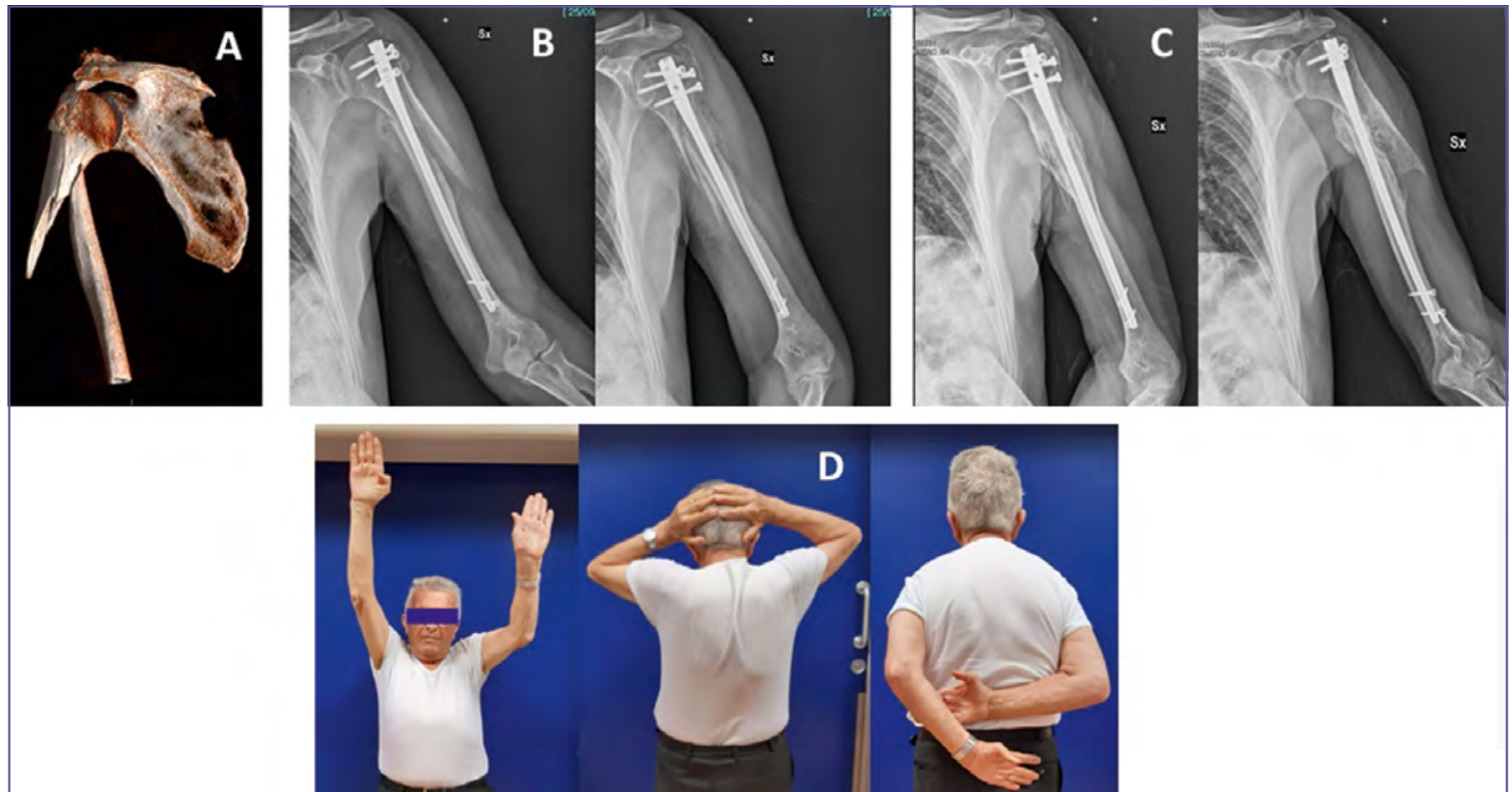

Figure 7. Clinical Case 7. AO $12 \mathrm{C} 1$ male 79y. Intramedullary nail fixation without anatomical reduction for a complex fracture. A) pre-operative CT-3D reconstruction; B) X-ray post-operative treatment at 1 month; C) X-ray 2 years after trauma show radiological healing; D) clinical photos 1 year after trauma show partial loss of function due to non-anatomical reduction and rotator cuff tear.

Shao et al. published one of the largest studies on humeral fractures in 2005 and found an incidence of radial nerve paralysis of $11.8 \%^{34}$.

Humeral shaft fractures associated with RNP are debilitating injuries and there is no clear consensus regarding if and when the nerve should be explored surgically. Radial nerve palsy can differ between primary or traumatic nerve injury and secondary or iatrogenic nerve injury.

Many authors have tried to identify a major cause for the incidence of radial nerve paralysis on humerus shaft fractures. Osterman et al. analyzed and compared their study with literature and identified that type $\mathrm{A}$ fractures are more often treated with intramedullary nail compared to type B and C fractures, which are usually treated with ORIF, with no significant difference in the time to onset of nerve recovery between fracture patterns and mean of treatment ${ }^{35}$. Streufert et al. examined their study and showed that the use of an anterolateral approach for middledistal third fractures led to a higher incidence of RNP than for proximal third fractures, likely due to the proximity of the nerve to the spiral groove and to its potentially difficult visualization and protection through an anterolateral approach.

Streufert et al. conclude that iatrogenic lesions of the radial nerve are not uncommon after surgical approach (12.2\%) and that, even if all surgical exposures are at risk, the approach used does not seem to significantly impact rates of iatrogenic RNP ${ }^{36}$. In case of low-energy trauma, primary radial nerve palsy is often caused by simple nerve contusion or stretching with the nerve being found usually macroscopically intact ${ }^{37,38}$. Early exploration of the radial nerve in primary traumatic palsy does not seem to be necessary, especially in closed fractures, where serious primary nerve damage requiring surgical repair is very rare. Earlier studies reported a high rate of spontaneous recovery in patients with primary nerve injury: a "wait and see" strategy seems to be widely accepted, recommending early nerve exploration only in special conditions, such as open fractures. If radial nerve palsy occurs with an open fracture of the humeral shaft, the nerve should be explored at the time of debridement of the wound ${ }^{39,40}$. Many other authors think that a "wait and observe" strategy for a potentially compressed or damaged radial nerve is wrong. Mangan et al. observed thant patients were treated with expectant management had an overall nerve recovery rate of $77.2 \%$. Failed expectant management and underwent late surgical exploration, which was defined as surgical intervention greater than 8 weeks postinjury, ultimately had a rate of recovery of only $68.1 \%$ (113/166). However, patients who underwent early surgical exploration, defined as surgical management within 3 weeks of injury, had a rate of radial nerve recovery of $89.8 \%$. The difference in the rate of radial nerve function return of those who underwent 


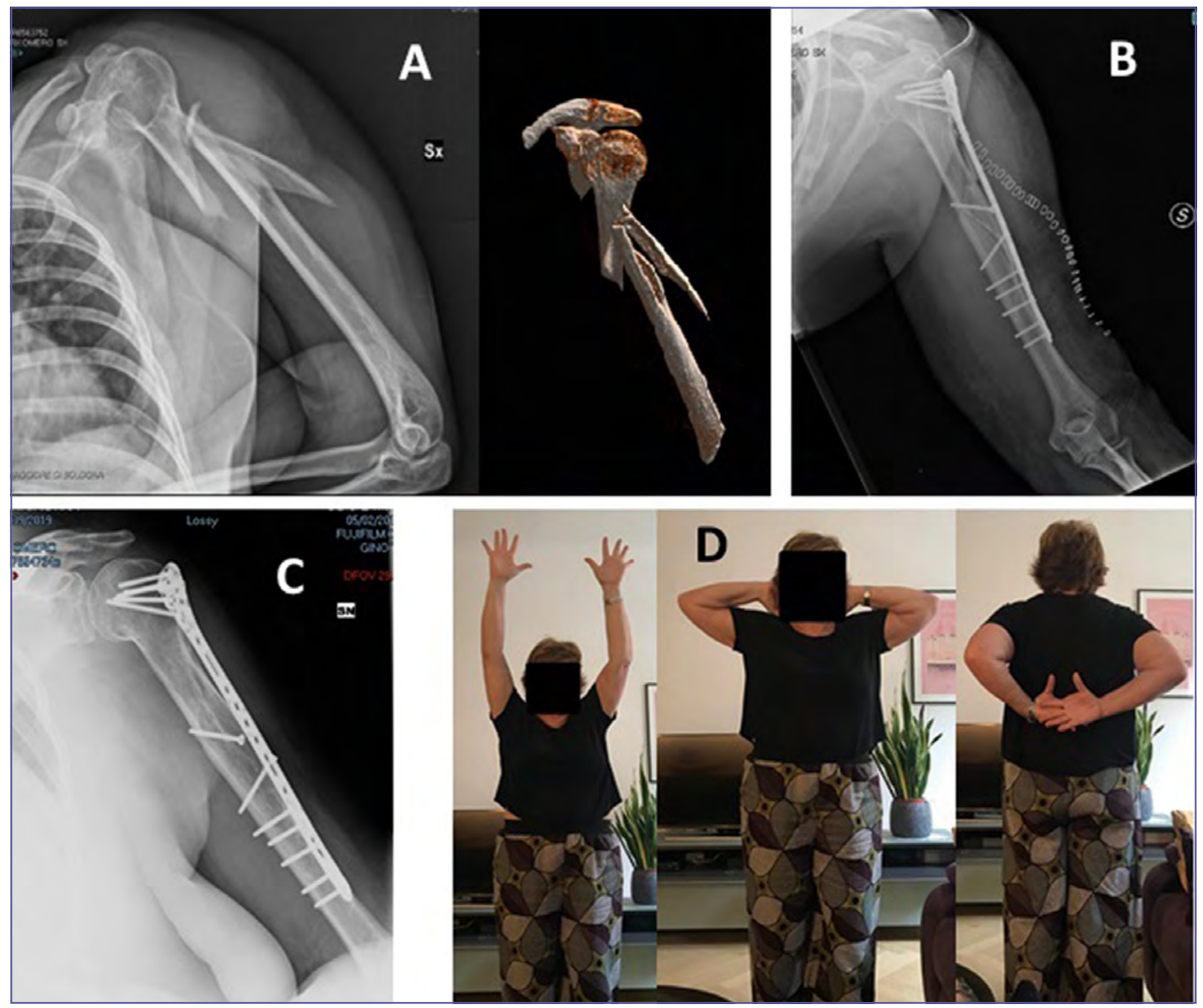

Figure 8. Clinical Case 8. AO 12C1 female 61y. ORIF with plate for complex fracture type C. A) pre-operative X-ray and CT-3D reconstruction; B) X-ray post-operative treatment; C) X-ray 18 months after trauma show radiological healing; D) clinical photos 18 months after trauma show very good functional recovery better than Clinical Case 7 due to a better anatomical reduction of the fracture. 


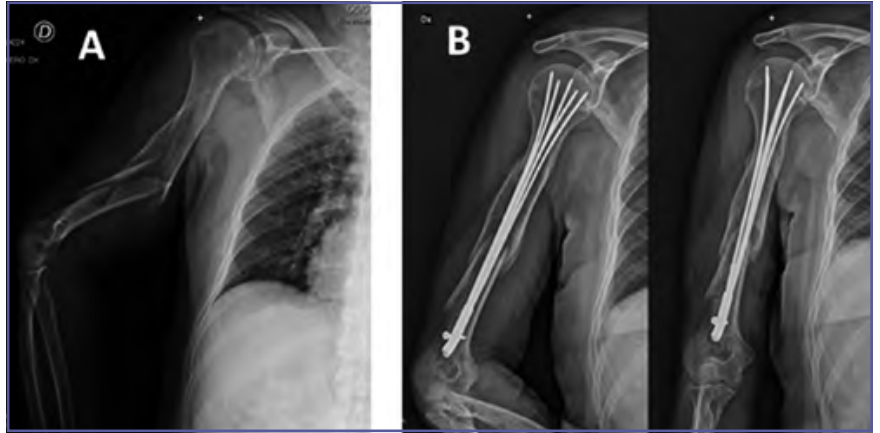

Figure 9. Clinical Case 9. AO 12C1 male 71y. Dynamic stabilization obtained with a retrograde elastic nail. $A$ ) pre-operative X-ray; B) X-ray 6 months after surgery.

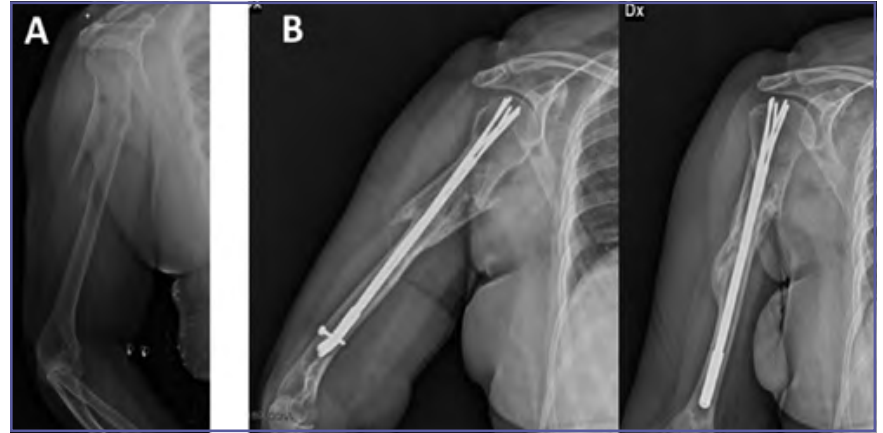

Figure 10. Clinical Case 10. AO 12C1 female 74y. Dynamic stabilization obtained with a retrograde elastic nail led its proximal migration in osteoporotic bone and it determined secondary rotator cuff lesions and subacromial impingement. A) pre-operative X-ray; B) X-ray 6 months after surgery.

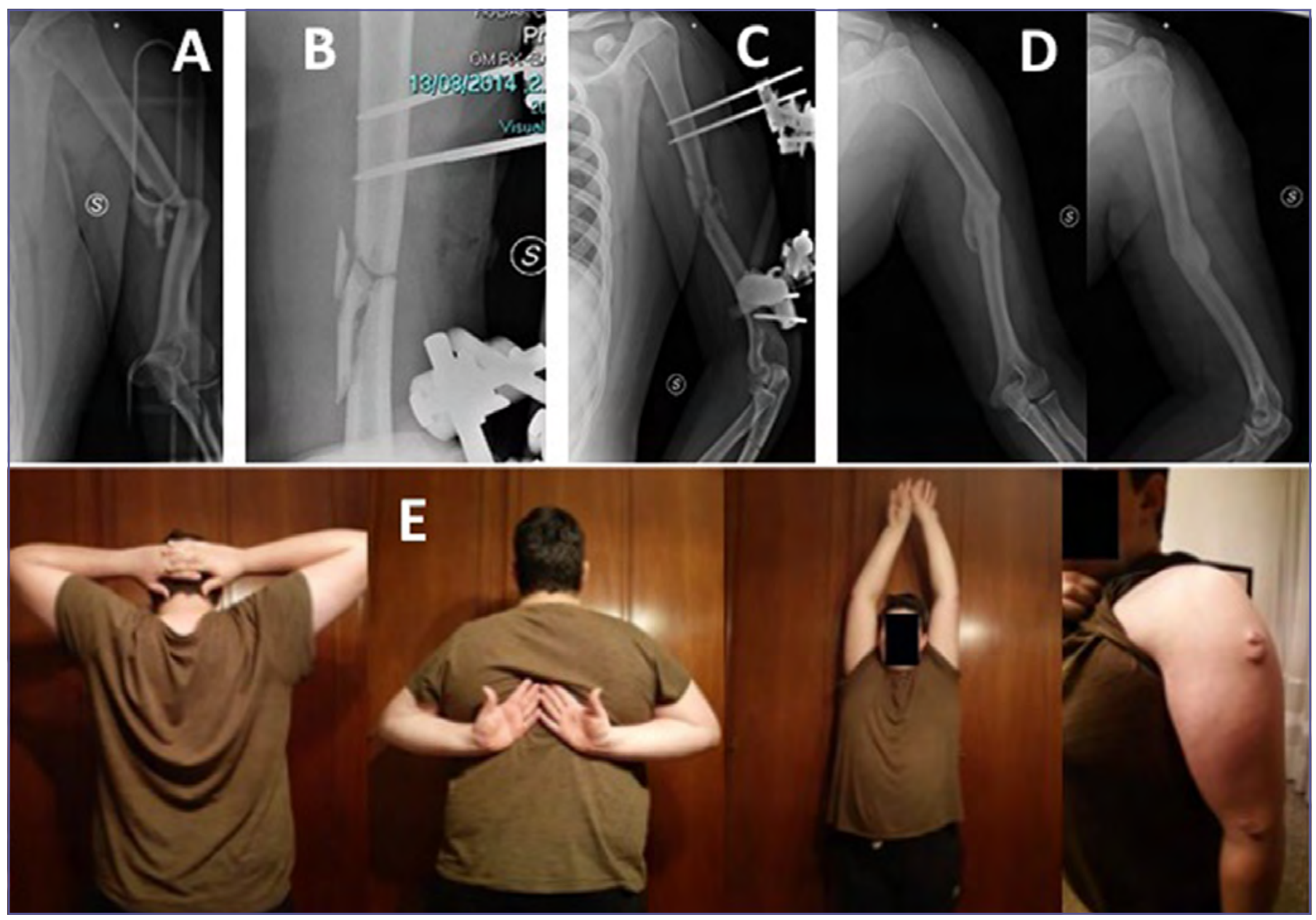

Figure 11. Clinical Case 11. AO 12B3 male 19y polytrauma. A) X-ray post-trauma; B) X-ray show urgent stabilization with external fixation; C) X-ray 1 month after trauma; D) X-ray 7 months after trauma; E) clinical photos show functional recovery of ROM 1 year after the trauma. 


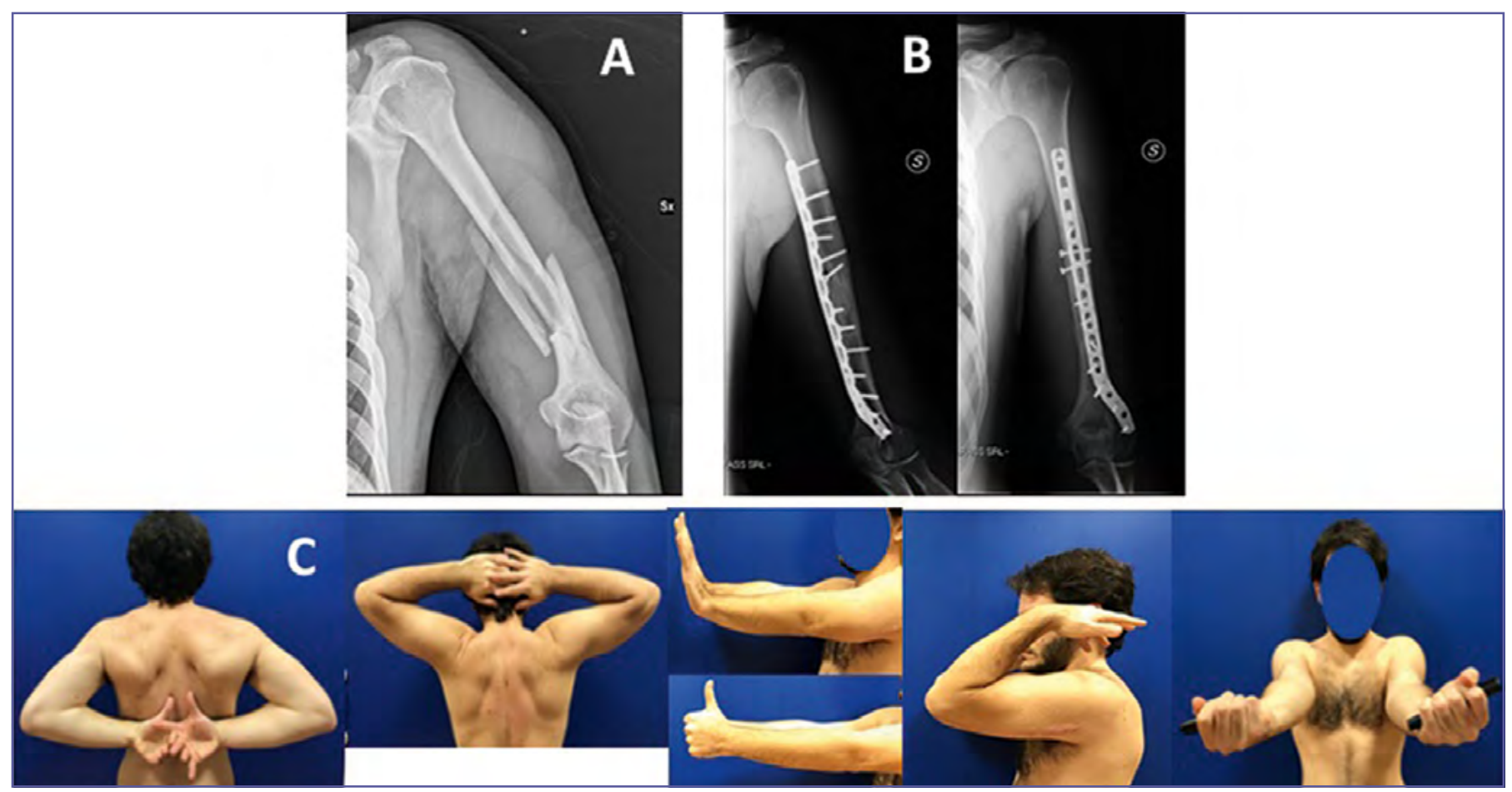

Figure 12. Clinical Case 12. AO 12B3 male 25y. Holsten-Lewis fracture with primary RNP, we adopted ORIF and exploration of the radial nerve. A) pre-operative X-ray; B) X-ray 1 year after trauma show radiological healing; C) clinical photos at 1 year show complete functional recovery.

early surgical exploration (89.8\%) compared with patients who underwent expectant management $(77.2 \%)$ alone was statistically significant ${ }^{41}$. For this reason Mangan et al. suggest that, for patients suffering from primary radial nerve palsies due to humeral shaft fractures, early exploration of the nerve within 3 weeks of injury. Diagnostic US can help differentiate patients who would benefit from early nerve exploration, nerve repair, or acute tendon transfers from patients expected to have spontaneous nerve recovery. Shao et al. ${ }^{3}$ published a systematic review in 2005 of radial nerve palsy associated with humeral shaft fractures and presented an algorithm to guide treatment. The algorithm includes US evaluation within three weeks of injury to assess the status of the radial nerve ${ }^{3}$. If the nerve is continuous, loss of function is thought to be secondary to neurapraxia which may be managed conservatively. If the nerve is lacerated or entrapped, early surgical intervention is preferred as nerve function would not be expected to recover spontaneously ${ }^{42}$. Rocchi et al. proposed in 2016 a diagnostic-therapeutic algorithm for the treatment of RNP in humerus shaft fractures. Surgical exploration could be initially deferred for fractures with low risk of radial nerve injury. The nerve palsy has to be followed up by neurophysiologic and clinic tests least 3 weeks after the trauma. If there are no electrophysiological changes at 6-12 weeks, a surgical exploration is recommended. The factors that determine the approach to nerve repair are the location and the duration of nerve injury ${ }^{43}$ (Figs. 12 A, B, C; Figs. 13A, B, C).

\section{Conclusions}

Humeral shaft fractures resulting from low-energy trauma were historically treated successfully with conservative methods ${ }^{8}$, but included a high percentage of nonunion, malunion, persistent radial nerve deficits and joint stiffness; indeed for these reasons surgical management has increased exponentially in recent years with many different techniques. Surgical treatment is usually reserved for when conservative treatment fails, irreducible fracture or unacceptable reduction, nonunion or malunion, for open fractures that require debridement and stabilization, in cases of neurovascular injury, in polytrauma, in fractures extended into the joints, in floating elbow and in patients who necessitate early mobilization. ORIF with plate allows anatomical reduction, direct viewing of the fractures site, interfragmentary compression and the possibility to explore and isolate the radial nerve. IMN is a valid alternative technique and it can be superior to ORIF in reducing blood loss, low incidence post-operative infections, low rate of nonunion and fast healing.

One of the most frequent complications of humeral shaft fractures is radial nerve palsy, which can be a debilitant injury and there is no clear existing consensus regarding if and when the nerve should be explored surgically. RNP for low energy trauma has to be followed up by neurophysiological and clinical 


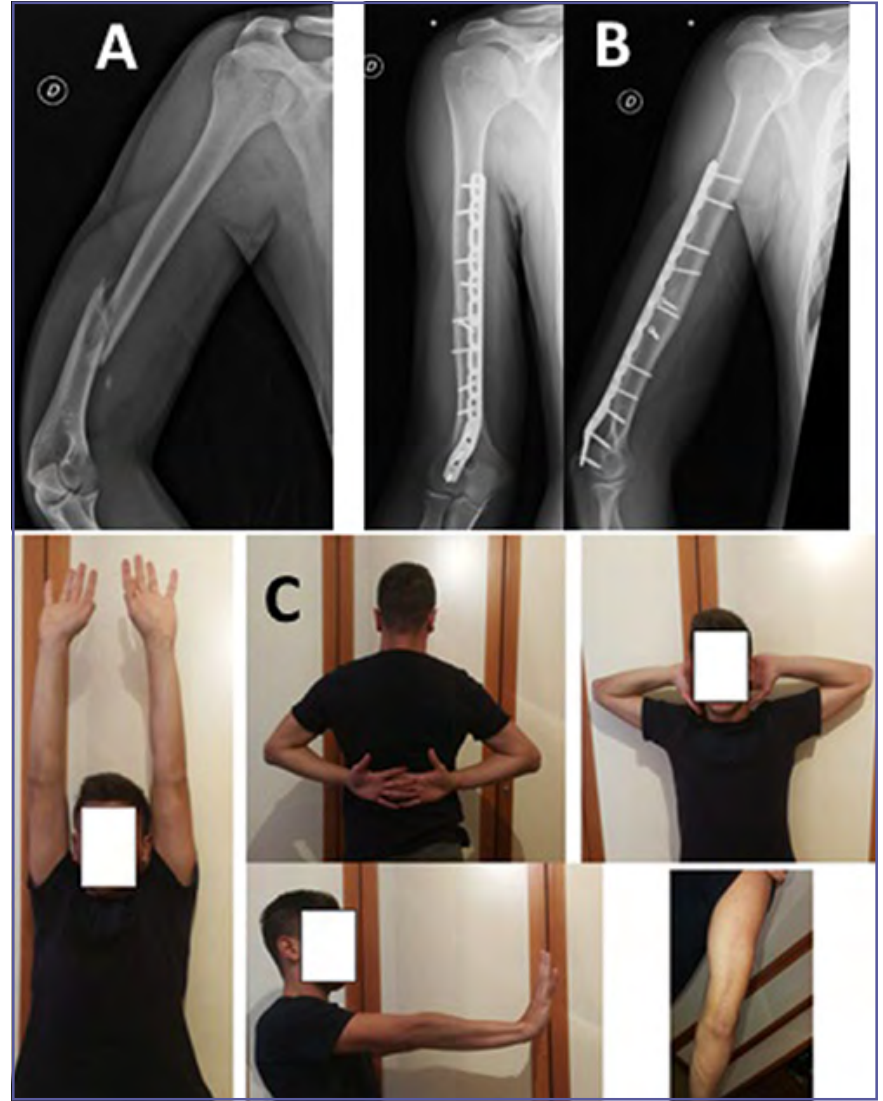

Figure 13. Clinical Case 13. AO $12 \mathrm{~A} 2$ male 21y. Case of secondary RNP restored after appropriate psychokinesis-therapy. A) pre-operative X-ray; B) X-ray 10 months after trauma shows radiological healing; $C$ ) clinical photos at $\mathbf{1 0}$ months show complete functional recovery.

tests at least at 3 weeks after the trauma; after 6-12 weeks of no nerve recovery a surgical exploration is recommended.

Although there are numerous randomized clinical trials and meta-analyses that have attempted to guide the surgeon in choosing, there is little evidence and no consensus as to the most suitable treatment. There is no specific algorithm for deciding since it is necessary it is necessary to consider many factors ${ }^{15}$. Treatment should be determined not only on the features of the fractures, but also on the experience of the surgeon and on patient compliance.

\section{References}

Updegrove GF, Mourad W, Abboud JA. Humeral shaft fractures. J Shoulder Elbow Surg 2018;27:e87-97. https://doi.org/10.1016/j. jse.2017.10.028

2 Ekholm R, Adami J, Tidermark J, et al. Fractures of the shaft of the humerus. An epidemiological study of 401 fractures. J Bone Joint Surg Br 2006;88:1469-73. https://doi.org/10.1302/0301-620X.88B11.17634
Tytherleigh-Strong G, Walls N, McQueen MM. The epidemiology of humeral shaft fractures. J Bone Joint Surg Br 1998;80:24953. https://doi.org/10.1302/0301-620x.80b2.8113

4 Bercik MJ, Tjoumakaris FP, Pepe M, et al. Humerus fractures at a regional trauma center: an epidemiologic study. Orthopedics 2013;36:e891-7. https://doi.org/10.3928/01477447-20130624-19 Humeral shaft - diagnosis - AO surgery reference (https://www2. aofoundation.org/wps/portal/!ut/p/a0/04_Sj9CPykssy0xPLMnMz0vMAfGjzOKN_A0M3D2DDbz9_UMMDRyDXQ3dw9wMDAzMjfULsh0VAbWjLW0!/?bone=Humerus\&segment=Shaft\&showPage $=$ diagnosis).

6 Balfour GW, Mooney V, Ashby ME. Diaphyseal fractures of the humerus treated with a ready-made fracture brace. J Bone Joint Surg Am 1982;64:11-3.

Ekholm R, Tidermark J, Törnkvist $\mathrm{H}$, et al. Outcome after closed functional treatment of humeral shaft fractures. J Orthop Trauma 2006;20:591-6. https://doi.org/10.1097/01. bot.0000246466.01287.04

8 Sarmiento A, Kinman PB, Galvin EG, et al. Functional bracing of fractures of the shaft of the humerus. J Bone Joint Surg Am 1977;59:596-601.

9 Papasoulis E1, Drosos GI, Ververidis AN, et al. Functional bracing of humeral shaft fractures. A review of clinical studies. Injury 2010;41:e21-7. https://doi.org/10.1016/j.injury.2009.05.004

10 Maresca A, Sangiovanni P, Cerbasi S, et al. Why a surgically treated humeral shaft fracture became a nonunion: review of 11 years in two trauma centers.Musculoskelet Surg 2017;101(Suppl 2):10512. https://doi.org/10.1007/s12306-017-0509-5

11 Ring D, Chin K, Taghinia AH, et al. Nonunion after functional brace treatment of diaphyseal humerus fractures. J Trauma 2007;62:11578. https://doi.org/10.1097/01.ta.0000222719.52619.2c

12 Shields E, Sundem L, Childs S, et al. The impact of residual angulation on patient reported functional outcome scores after non-operative treatment for humeral shaft fractures. Injury 2016;47:9148. https://doi.org/10.1016/j.injury.2015.12.014

13 Harkin FE, Large RJ. Humeral shaft fractures: union outcomes in a large cohort. J Shoulder Elbow Surg 2017;26:1881-8. https:// doi.org/10.1016/j.jse.2017.07.001

14 Koch PP1, Gross DF, Gerber C. The results of functional (Sarmiento) bracing of humeral shaft fractures. J Shoulder Elbow Surg 2002;11:143-50. https://doi.org/10.1067/mse.2002.121634

15 Bisaccia M, Meccariello L, Rinonapoli G, et al. Comparison of plate, nail and external fixation in the management of diaphyseal fractures of the humerus. Med Arch 2017;71:97-102. https://doi.org/10.5455/ medarh.2017.71.97-102

16 Westrick E, Hamilton B, Toogood P, et al. Humeral shaft fractures: results of operative and non-operative treatment. Int Orthop 2017;41:385-95. https://doi.org/10.1007/s00264-016-3210-7

17 Capo JT, Criner KT, Shamian B. Exposures of the humerus for fracture fixation. Hand Clin 2014;30:401-14. https://doi. org/10.1016/j.hcl.2014.07.001

18 Zlotolow DA, Catalano LW, Barron OA, et al. Surgical exposures of the humerus. J Am Acad Orthop Surg 2006;14:754-65. https:// doi.org/10.5435/00124635-200612000-00007

19 Rocchi M, Tarallo L, Mugnai R, et al. Humerus shaft fracture complicated by radial nerve palsy: is surgical exploration necessary?. Musculoskelet Surg 2016;100 (Suppl 1):53-60. https://doi. org/10.1007/s12306-016-0414-3

20 Carl D, Pratt J, Patterson JMM, et al. The radial nerve in the bra- 
chium: an anatomic study in human cadavers. J Hand Surg Am 2007;32:1177-82. https://doi.org/10.1016/j.jhsa.2006.07.001

21 Korompilias AV, Lykissa MG, Kostas-Agnantis IP, et al. Approach to radial nerve palsy caused by humerus shaft fracture: is primary exploration necessary? Injury 2013;44:323-6. https://doi. org/10.1016/j.injury.2013.01.004

22 Chang G, Ilyas AM. Radial nerve palsy after humeral shaft fractures: the case for early exploration and a new classification to guide treatment and prognosis. Hand Clin 2018;34:105-12. https://doi.org/10.1016/j.hcl.2017.09.011

23 Dabezies EJ, Banta CJ, Murphy CP, et al. Plate fixation of the humeral shaft for acute fractures, with and without radial nerve injuries. J Orthop Trauma 1992;6:10-3.

24 Seigerman DA, Choung EW, Yoon RS, et al. Identification of the radial nerve during the posterior approach to the humerus: a cadaveric study. J Orthop Trauma 2012;26:226-8. https://doi. org/10.1097/BOT.0b013e31821d0200

25 Gerwin M, Hotchkiss RN, Weiland AJ. Alternative operative exposures of the posterior aspect of the humeral diaphysis with reference to the radial nerve. J Bone Joint Surg Am 1996;78:1690-5. https://doi.org/10.2106/00004623-199611000-00008

26 Ma J, Xing D, Ma X, et al. Intramedullary nail versus dynamic compression plate fixation in treating humeral shaft fractures: grading the evidence through a meta-analysis. PLoS One 2013;8:e82075. https://doi.org/10.1371/journal.pone.0082075

27 Wen $\mathrm{H}$, Zhu S, Li C, et al. Antegrade intramedullary nail versus plate fixation in the treatment of humeral shaft fractures: an update meta-analysis. Medicine (Baltimore) 2019;98:e17952. https://doi.org/10.1097/MD.0000000000017952

28 Zhao J-G, Wang J, Wang C, et al. Intramedullary nail versus plate fixation for humeral shaft fractures: a systematic review of overlapping meta-analyses. Medicine (Baltimore) 2015;94:e599. https://doi.org/10.1097/MD.0000000000000599

29 Verdano MA, Pellegrini A, Schiavi P, et al. Humeral shaft fractures treated with antegrade intramedullary nailing: what are the consequences for the rotator cuff? Int Orthop 2013;37:2001-7. https://doi.org/10.1007/s00264-013-2007-1

30 O'Donnell TMP, McKenna JV, Kenny P, et al. Concomitant injuries to the ipsilateral shoulder in patients with a fracture of the diaphysis of the humerus. J Bone Joint Surg Br 2008;90:61-5. https:// doi.org/10.1302/0301-620X.90B1.19215
31 Sala F, Chiodini F, Baù D, et al. Closed humeral shaft fractures treated by elastic intramedullary retrograde nail. Chir Organi Mov 2002;87:163-8.

32 Basso M, Formica , Cavagnaro L, et al. Unilateral external fixator in the treatment of humeral shaft fractures: results of a single center retrospective study. Musculoskelet Surg 2017;101:237-42. https://doi.org/10.1007/s12306-017-0473-0

33 Schwab TR, Stillhard PF, Schibli S, et al. Radial nerve palsy in humeral shaft fractures with internal fixation: analysis of management and outcome. Eur J Trauma Emerg Surg 2018;44:235-43.

34 ÈShao YC, Harwood P, Grotz MR, et al. Radial nerve palsy associated with fractures of the shaft of the humerus: a systematic review. J Bone Jt Surg Br 2005;87:1647-52.

35 Ostermann RC, Lang NW, Joestl J, et al. Fractures of the humeral shaft with primary radial nerve palsy: do injury mechanism, fracture type, or treatment influence nerve recovery?. J Clin Med 2019;8:1969. https://doi.org/10.3390/jcm8111969

36 Streufert BD, Eaford I, Sellers TR, et al. Iatrogenic nerve palsy occurs with anterior and posterior approaches for humeral shaft fixation. J Orthop Trauma 2020;34:163-8. https:/doi.org/10.1097/ BOT.0000000000001658

37 Ekholm R, Ponzer S, Tornkvist H, et al. Primary radial nerve palsy in patients with acute humeral shaft fractures. J Orthop Trauma 2008;22:408-14.

38 Venouziou AI, Dailiana ZH, Varitimidis SE, et al. Radial nerve palsy associated with humeral shaft fracture. Is the energy of trauma a prognostic factor? Injury 2011;42:1289-93.

39 DeFranco MJ, Lawton JN. Radial nerve injuries associated with humeral fractures. J Hand Surg 2006;31:655-63.

40 Prodromo J, Goitz RJ. Management of radial nerve palsy associated with humerus fracture. J Hand Surg 2013;38:995-8.

41 Mangan JJ, Graham J, Ilyas AM. Radial nerve palsy recovery with fractures of the humerus. J Am Acad Orthop Surg 2020;28:e2639. https://doi.org/10.5435/JAAOS-D-18-00142

42 Esparza M, Wild JR, Minnock C, et al. Ultrasound evaluation of radial nerve palsy associated with humeral shaft fractures to guide operative versus non-operative treatment. Acta Med Acad 2019;48:183-92. https://doi.org/10.5644/ama2006-124.257

43 Rocchi M, Tarallo L, Mugnai R, et al. Humerus shaft fracture complicated by radial nerve palsy: is surgical exploration necessary? Musculoskelet Surg 2016;100(Suppl 1):53-60. 